\title{
Geometrical Method for Thermal Instability of Nonlinearly Charged BTZ Black Holes
}

\author{
Seyed Hossein Hendi, ${ }^{1,2}$ Shahram Panahiyan, ${ }^{1}$ and Behzad Eslam Panah ${ }^{1}$ \\ ${ }^{1}$ Physics Department and Biruni Observatory, College of Sciences, Shiraz University, Shiraz 71454, Iran \\ ${ }^{2}$ Research Institute for Astronomy and Astrophysics of Maragha (RIAAM), P.O. Box 55134-441, Maragha, Iran \\ Correspondence should be addressed to Seyed Hossein Hendi; hendi@shirazu.ac.ir
}

Received 20 April 2015; Revised 29 June 2015; Accepted 6 July 2015

Academic Editor: George Siopsis

Copyright (c) 2015 Seyed Hossein Hendi et al. This is an open access article distributed under the Creative Commons Attribution License, which permits unrestricted use, distribution, and reproduction in any medium, provided the original work is properly cited. The publication of this article was funded by $\mathrm{SCOAP}^{3}$.

\begin{abstract}
We consider three-dimensional BTZ black holes with three models of nonlinear electrodynamics as source. Calculating heat capacity, we study the stability and phase transitions of these black holes. We show that Maxwell, logarithmic, and exponential theories yield only type one phase transition which is related to the root(s) of heat capacity, whereas, for correction form of nonlinear electrodynamics, heat capacity contains two roots and one divergence point. Next, we use geometrical approach for studying classical thermodynamical behavior of the system. We show that Weinhold and Ruppeiner metrics fail to provide fruitful results and the consequences of the Quevedo approach are not completely matched to the heat capacity results. Then, we employ a new metric for solving this problem. We show that this approach is successful and all divergencies of its Ricci scalar and phase transition points coincide. We also show that there is no phase transition for uncharged BTZ black holes.
\end{abstract}

\section{Introduction}

One of the interesting subjects for recent gravitational studies is the investigation of three-dimensional black holes [1-9]. Considering three-dimensional solutions helps us to find a profound insight in the black hole physics, quantum view of gravity, and also its relations to string theory [10-14]. Moreover, three-dimensional spacetimes play an essential role in improving our understanding of gravitational interaction in low dimensional manifolds [15]. Due to these facts, some of physicists have an interest in the $(2+1)$-dimensional manifolds and their attractive properties [16-27].

The Maxwell theory is in agreement with experimental results, but it fails regarding some important issues such as self-energy of point-like charges which motivates one to regard nonlinear electrodynamics (NED). There are some evidences that motivate one to consider NED theories: solving the problem of point-like charge self-energy, understanding the nature of different complex systems, obtaining more information and insight regarding quantum gravity, compatible with AdS/CFT correspondence and string theory frames, description of pair creation for Hawking radiation, and the behavior of the compact astrophysical objects such as neutron stars and pulsars [28-30]. Therefore, many authors investigated the black hole solutions with nonlinear sources [31-49].

On the other hand, thermodynamical structure of the black holes has been of great interest. It is due to the fact that, according to AdS/CFT correspondence, black hole thermodynamics provides a machinery to map a solution in AdS spacetime to a conformal field on the boundary of this spacetime [13-15]. Also it was recently pointed out that considering cosmological constant as a thermodynamical variable leads to the behavior similar to the Van Der Waals liquid/gas system [50-57]. In addition, phase transition of the black holes plays an important role in exploring the critical behavior of the system near critical points. There are several approaches that one can employ to study the phase transition. One of these approaches is studying the behavior of the heat capacity. It is argued that roots and divergence points of the heat capacity are representing two types of phase transition [58-64]. In addition, studying the heat capacity and its behavior enables one to study the thermal stability of the black holes [65-70]. 
Another approach for studying phase transition of black holes is through thermodynamical geometry. The concept is to construct a spacetime by employing the thermodynamical properties of the system. Then, by studying the divergence points of thermodynamical Ricci scalar of the metric, one can investigate phase transition points. In other words, it is expected that divergencies of thermodynamical Ricci scalar (TRS) coincide with phase transition points of the black holes. Firstly, Weinhold introduced differential geometric concepts into ordinary thermodynamics [71, 72]. He considered a kind of metric defined as the second derivatives of internal energy with respect to entropy and other extensive quantities for a thermodynamical system. Later Ruppeiner [73, 74] introduced another metric and defined the minus second derivatives of entropy with respect to the internal energy and other extensive quantities. The Ruppeiner metric is conformal to the Weinhold metric with the inverse temperature as the conformal factor. It is notable that both metrics have been applied to study the thermodynamical geometry of ordinary systems [75-80]. In particular, it was found that the Ruppeiner geometry carries information of phase structure of thermodynamical system. For the systems with no statistical mechanical interactions (e.g., ideal gas), the scalar curvature is zero and the Ruppeiner metric is flat. Because of the success of their applications to ordinary thermodynamical systems, they have also been used to study black hole phase structures and lots of results have been obtained for different types of black holes [81-86].

It is notable that these two approaches fail in order to describe phase transition of several black holes [87]. In order to overcome this problem, Quevedo proposed new types of metrics for studying geometrical structure of the black hole thermodynamics [88, 89]. This method was employed to study the geometrical structure of the phase transition of the black holes [58-64, 90-94] and proved to be a strong machinery for describing phase transition of the black holes. But the results of this approach was not completely coincided with the results of classical thermodynamics arisen from the heat capacity [87]. In [87], a new metric was proposed in which the denominator of its Ricci scalar is only constructed of numerator and denominator of the heat capacity. Several phase transitions of the black holes have been studied in context of the HPEM (Hendi-Panahiyan-Eslam PanahMomennia) metric [87].

In this paper, we study thermal stability and phase transition of the BTZ black holes in the presence of several NED models in context of heat capacity. Then, we employ
Weinhold, Ruppeiner, and Quevedo methods for studying geometrothermodynamics of these black holes. We will see that Weinhold and Ruppeiner metrics fail to provide fruitful results and the consequences of the Quevedo approach are not completely matched to the heat capacity results. Then, we employ the HPEM metric and study the phase transition of these black holes in context of geometrothermodynamics. We end the paper with some closing remarks.

\section{Nonlinearly Charged BTZ Black Hole Solutions}

The $(2+1)$-dimensional action of Einstein gravity with NED field in the presence of cosmological constant is given by

$$
I=-\frac{1}{16 \pi} \int d^{3} x \sqrt{-g}[R-2 \Lambda+L(\mathscr{F})],
$$

where $R$ is the Ricci scalar and the cosmological constant is $\Lambda=-l^{-2}$ in which $l$ is a scale factor. Also $L(F)$ is the Lagrangian of NED, in which we consider three models. First model was proposed by Hendi (HNED) [95], second one is Soleng theory (SNED) [96], and third one is correction form of NED (CNED) [69]:

$$
L(\mathscr{F})= \begin{cases}\beta^{2}\left(\exp \left(-\frac{\mathscr{F}}{\beta^{2}}\right)-1\right), & \text { HNED, } \\ -8 \beta^{2} \ln \left(1+\frac{\mathscr{F}}{8 \beta^{2}}\right), & \text { SNED, } \\ -\mathscr{F}+\alpha \mathscr{F}^{2}+\mathcal{O}\left(\alpha^{2}\right), & \text { CNED, }\end{cases}
$$

where $\beta$ and $\alpha$ are called the nonlinearity parameters, the Maxwell invariant $\mathscr{F}=F_{\mu \nu} F^{\mu \nu}$ in which $F_{\mu \nu}=\partial_{\mu} A_{\nu}-$ $\partial_{\nu} A_{\mu}$ is the electromagnetic field tensor, and $A_{\mu}$ is the gauge potential. We should note that for $\beta \rightarrow \infty$ (HNED and SNED branches) and $\alpha \rightarrow 0$ (CNED branch) the Maxwell Lagrangian can be recovered.

The nonlinearly charged static black hole solutions can be introduced with the following line element:

$$
d s^{2}=-f(r) d t^{2}+\frac{d r^{2}}{f(r)}+r^{2} d \theta^{2}
$$

where the metric function $f(r)$ was obtained in $[70,95]$

$$
f(r)=\frac{r^{2}}{l^{2}}-m+ \begin{cases}\frac{\beta r q\left(1-2 L_{W}\right)}{\sqrt{L_{W}}}-\frac{\beta^{2} r^{2}}{2}+q^{2}\left[\ln \left(\frac{\beta^{2} l^{2}}{2 q^{2}}\right)-E i\left(1, \frac{L_{W}}{2}\right)-\gamma+3\right], & \text { HNED, } \\ 4 \beta^{2} r^{2}\left[\ln \left(\frac{\Gamma+1}{2}\right)+3\right]-q^{2}\left[\ln \left(\frac{\beta^{2} r^{4}(\Gamma-1)(\Gamma+1)^{3}}{4 q^{2} l^{2}}\right)+\frac{6}{\Gamma-1}-2\right], & \text { SNED, } \\ -2 q^{2} \ln \left(\frac{r}{l}\right)-\frac{2 \alpha q^{4}}{r^{2}}+\mathcal{O}\left(\alpha^{2}\right), & \text { CNED, }\end{cases}
$$


where $\Gamma=\sqrt{1+q^{2} / r^{2} \beta^{2}}$ and $m$ and $q$ are integrations constant which are related to mass and the electric charge of the black hole, respectively. In addition, $L_{W}=$ Lambert $W\left(4 q^{2} / \beta^{2} r^{2}\right), \gamma=\gamma(0) \simeq 0.57722$, and the special function $\operatorname{Ei}(1, x)=\int_{1}^{\infty}\left(e^{-x z} / z\right) d z$.

The entropy and the electric charge of the obtained NED black hole solutions can be calculated with the following forms $[70,95]$ :

$$
S=\frac{\pi r_{+}}{2}
$$

$$
Q=\frac{\pi q}{2}
$$

where $r_{+}$is the event horizon of black hole. On the other hand, the quasilocal mass, which is related to geometrical mass, can be obtained as $[70,95]$

$$
M=\frac{\pi}{8} m
$$

Regarding (5) with (6) and obtaining $m$ by using $f(r=$ $\left.r_{+}\right)=0$, for these three cases of BTZ black holes, we can write

$$
M=\frac{1}{8 \pi} \times \begin{cases}\frac{4 \beta S Q\left(1-2 L_{W}^{\prime}\right)}{\sqrt{L_{W}^{\prime}}}-\frac{2 S^{2}\left(l^{2} \beta^{2}-2\right)}{l^{2}}+4 Q^{2}\left[\ln \left(\frac{\beta^{2} l^{2} \pi^{2}}{Q^{2}}\right)-E i\left(1, \frac{L_{W}^{\prime}}{2}\right)-\gamma+3\right], & \text { HNED } \\ \frac{4 S^{2}}{l^{2}}+16 \beta^{2} S^{2}\left[\ln \left(\frac{\Gamma^{\prime}+1}{2}\right)+3\right]-4 Q^{2}\left[\ln \left(\frac{\beta^{2} S^{4}\left(\Gamma^{\prime}-1\right)\left(\Gamma^{\prime}+1\right)^{3}}{Q^{2} l^{2} \pi^{2}}\right)+\frac{6}{\Gamma^{\prime}-1}-2\right], & \text { SNED, } \\ \frac{4 S^{2}}{l^{2}}-8 Q^{2} \ln \left(\frac{2 S}{l \pi}\right)-\frac{8 Q^{4}}{S^{2}} \alpha, & \text { CNED },\end{cases}
$$

where $\Gamma^{\prime}=\sqrt{1+Q^{2} / S^{2} \beta^{2}}$ and $L_{W}^{\prime}=\operatorname{Lambert} W\left(4 Q^{2} / \beta^{2} S^{2}\right)$. Having conserved and thermodynamic quantities at hand, it was shown that the first law of thermodynamics may be satisfied [95]. The main goal of this paper is investigating thermal stability and phase transition for these black holes.

2.1. Heat Capacity. In order to investigate thermal stability and phase transition, one can usually adopt two different approaches to the matter at hand. In one method, the electric charge is considered as a fixed parameter and heat capacity of the black hole will be calculated. The positivity of the heat capacity is sufficient to ensure the local thermal stability of the solutions and its divergencies are corresponding to the phase transition points. This approach is known as canonical ensemble. Another approach for studying thermal stability of the black holes is grand canonical ensemble. In this approach, thermal stability is investigated by calculating the determinant of Hessian matrix of $M(S, Q)$ with respect to its extensive variables. The positivity of this determinant also represents the local stability of the solutions. Although these two approaches are different fundamentally, one expects the results to be the same for both ensembles; that is, ensemble independent. Here we use the first method for studying thermal stability. For this purpose, the system is considered to be in fixed charge and the heat capacity has the following form:

$$
C_{Q}=\left(\frac{\partial M}{\partial S}\right)_{Q}\left(\frac{\partial^{2} M}{\partial S^{2}}\right)_{Q}^{-1}
$$

It is notable that when we study heat capacity for investigating the phase transition, we encounter with two different phenomena. In one, the changes in the signature of the heat capacity are representing a phase transition of the system. In other words, if the heat capacity is negative, then the system is in thermally unstable phase, whereas, for the case of the positive $C_{Q}$, the system is thermally stable. The roots of the heat capacity in this case are representing phase transition points which means one should solve the following equation:

$$
\left(\frac{\partial M}{\partial S}\right)_{Q}=0
$$

where hereafter we call this type of the phase transition as type one. It is a matter of calculation to show that

$$
\begin{aligned}
& \left(\frac{\partial M}{\partial S}\right)_{Q} \\
& =\left\{\begin{array}{l}
-\frac{2 l^{2} Q^{2} e^{(1 / 2) L_{W}^{\prime}} \sqrt{L_{W}^{\prime}}+S\left[S\left(\beta^{2} l^{2}-2\right) \sqrt{L_{W}^{\prime}}\left(1+L_{W}^{\prime 2}\right)+Q \beta l^{2} L_{W}^{\prime}\left(2 L_{W}^{\prime}-1\right)+2 Q \beta l^{2}\right]}{2 \pi S l^{2} \sqrt{L_{W}^{\prime}}\left(1+L_{W}^{\prime}\right)} \\
\frac{4 \beta^{2} l^{2} Q^{2}\left(Q^{2}-S^{2} \beta^{2}\right) \ln \left(\left(1+\Gamma^{\prime}\right) / 2\right)+8 S^{2} Q^{2} l^{2} \beta^{4}+Q^{4}\left(1+S^{2}+8 \beta^{2} l^{2}\right)}{2 \pi S^{3} \beta^{4} l^{2} \Gamma^{\prime}\left(1-\Gamma^{\prime}\right)\left(1-\Gamma^{\prime 2}\right)}+\frac{S^{4} \beta^{2}\left(1-S^{2} \sqrt{\Gamma^{\prime}}\right)-6 Q^{4} l^{2}}{2 \pi S^{3} \beta^{2} l^{2}\left(1-\Gamma^{\prime}\right)\left(1-\Gamma^{\prime 2}\right)}+\frac{2 S^{2} \ln \left(\left(\Gamma^{\prime}+1\right) / 2\right)+6 \beta^{2}+Q^{2}}{\pi S\left(1-\Gamma^{\prime}\right)}, \quad \mathrm{SNED}, \\
\frac{\left(2 Q^{4} \alpha-S^{2} Q^{2}\right) l^{2}+S^{4}}{\pi l^{2} S^{2}},
\end{array}\right. \text { HNED, }
\end{aligned}
$$


The other case of phase transition is the divergency of the heat capacity. In other words, the singular points of the heat capacity are representing places in which system goes under phase transition. This assumption leads to the fact that the roots of the denominator of the heat capacity are representing phase transitions. Therefore, we have the following relation for this type of phase transition:

$$
\left(\frac{\partial^{2} M}{\partial S^{2}}\right)_{Q}=0
$$

where we call this type of the phase transition as type two. Due to economical reasons, we did not write the explicit relations of $\left(\partial^{2} M / \partial S^{2}\right)_{Q}$ for different BTZ solutions.

2.2. Geometrical Thermodynamics. In order to have an effective geometrical approach for studying phase transition of a system, one can build a suitable thermodynamical metric and investigate its Ricci scalar. Thermodynamical metrics were introduced based on the Hessian matrix of the mass (internal energy) with respect to the extensive variables. Therefore, although the electric charge is a fixed parameter for calculating the heat capacity in canonical ensemble, it may be an extensive variable for constructing thermodynamical metrics. In this method we expect that TRS diverges in both types of the mentioned phase transition points. In other words, the denominator of TRS must be constructed in a way that contains roots of the denominator and numerator of the heat capacity. In what follows, we will study the denominator of TRS of the several geometrical approaches and follow the recently proposed thermodynamical metric whose denominator only contains numerator and denominator of the heat capacity and, therefore, divergencies of TRS coincide with roots and divergences of the heat capacity.

In order to find the roots and divergence points of heat capacity, we should solve its numerator and denominator, separately. Solving the mentioned equations with respect to entropy leads to

$$
\left.S_{0} \equiv S\right|_{C_{Q}=0}= \begin{cases}\frac{4 Q \beta l^{2} L \omega}{\left(2-\beta^{2} l^{2}\right) \sqrt{1+2 L \omega}}, & \text { HNED, } \\ \frac{Q}{\beta \sqrt{L \omega(2+L \omega)}}, & \text { SNED, } \\ \frac{Q}{2} \sqrt{ \pm 2 l\left(\sqrt{l^{2}-8 \alpha} \pm l\right)}, & \text { CNED }\end{cases}
$$

$$
\begin{aligned}
& \left.S_{\infty} \equiv S\right|_{C_{Q} \rightarrow \infty} \\
& = \begin{cases}\frac{2 Q \beta l^{2}}{\left(\beta^{2} l^{2}-2\right) \sqrt{2 \ln \left(1-2 / \beta^{2} l^{2}\right)}}, & \text { HNED, } \\
\frac{Q}{2 \beta \sqrt{\exp \left(-1 / 4 \beta^{2} l^{2}\right)\left[\exp \left(-1 / 4 \beta^{2} l^{2}\right)-1\right]}}, & \text { SNED, } \\
\frac{Q}{2} \sqrt{2 l\left(\sqrt{l^{2}+24 \alpha}-l\right)}, & \text { CNED, }\end{cases}
\end{aligned}
$$

where

$$
\begin{aligned}
& L \omega=\operatorname{Lambert} W\left[-\frac{\left(\beta^{2} l^{2}-2\right)}{2 \beta^{2} l^{2} e^{1 / 2}}\right], \\
& L \omega=\operatorname{Lambert} W\left[-2 \exp \left(-\frac{8 \beta^{2} l^{2}+1}{4 \beta^{2} l^{2}}\right)\right] .
\end{aligned}
$$

It is evident from obtained equation for HNED and SNED that there is only one real positive entropy in which heat capacity vanishes. Interestingly, in case of CNED theory, we find two roots for heat capacity. It is evident that the roots are increasing functions of the electric charge in these theories. As for nonlinearity parameter, in case of the HNED and SNED theories, the root is an increasing function of $\beta$. For CNED theory, the smaller root is an increasing function of $\alpha$, whereas the larger root is a decreasing function of it.

Now we are in a position to study the existence of the type two phase transition point which is related to divergency of the heat capacity. Considering HNED branch of (13), one finds that $S_{\infty}$ is not real for all values of $l$ and $\beta$. Therefore, there is no physical divergence point for the heat capacity of HNED model. Next, for the case of SNED model, the same behavior is seen. In other words, since $0 \leq \exp (-1 / x)<1$ for $0 \leq x<\infty$, we cannot obtain real $S_{\infty}$ for all values of $l$ and $\beta$. Next, we should investigate CNED model. Regarding (13), we find that there is a divergence point for heat capacity (we should note that in this paper we consider positive $\alpha$ ). In other words, this theory of nonlinear electromagnetic field enjoys the phase transition of type two. The divergence point is an increasing function of the nonlinearity and electric charge parameters. It is worthwhile to mention that, for the case of vanishing nonlinearity parameter in this theory, $S_{\infty}$ goes to zero. In other words, there is no divergence point for heat capacity of Maxwell theory. It is also clear that, in case of chargeless BTZ black holes, there is no root and divergence point for heat capacity. In other words, in case of chargeless BTZ black holes, there is not any kind of phase transition.

2.2.1. Weinhold and Ruppeiner Metrics. The Weinhold metric was given in $[71,72]$

$$
d S_{W}^{2}=g_{a b}^{W} d X^{a} d X^{b}
$$

where $g_{a b}^{W}=\partial^{2} M\left(X^{c}\right) / \partial X^{a} \partial X^{b}$ and also $X^{a} \equiv X^{a}\left(S, N^{i}\right)$, where $N^{i}$ denotes other extensive variables of the system. In case of Weinhold approach, one is considering the mass of the system as potential, other parameters such as entropy and electric charge as extensive parameters and related quantities such as temperature, and electric potential as intensive parameters.

The Ruppeiner metric was defined as $[73,74]$

$$
d S_{R}^{2}=g_{a b}^{R} d X^{a} d X^{b}
$$

where $g_{a b}^{R}=-\partial^{2} S\left(X^{c}\right) / \partial X^{a} \partial X^{b}$ and $X^{a} \equiv X^{a}\left(M, N^{i}\right)$. In this case, the thermodynamical potential is entropy. It is worthwhile to mention that, according to the proposal of 

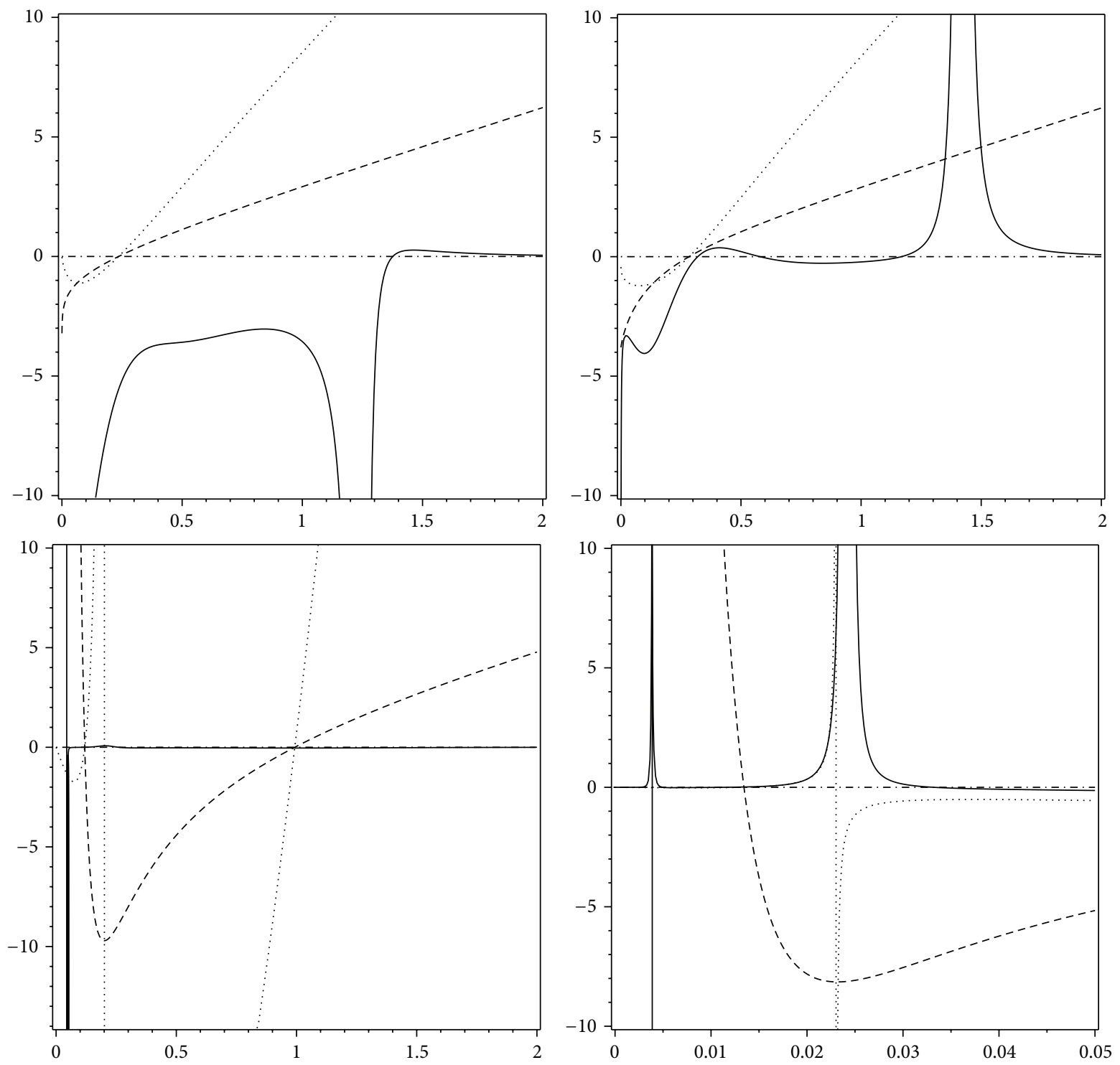

FIGURE 1: Weinhold Ricci scalar (solid line), heat capacity (dotted line), and temperature (dashed line) versus $S$ for $l=1$. HNED model (upleft panel) and SNED model (up-right panel): $q=0.3, \beta=1$. CNED model (down-left panel) and CNED model (down-right panel): $q=1$, $\alpha=0.007$ (different scales).

the Quevedo, these two approaches are related to each other by a Legendre transformation [97].

Taking into account thermodynamical metrics of Weinhold and Ruppeiner, one can obtain their Ricci scalars. Since we would like to investigate divergence points of TRS, $\mathscr{R}$, we focus on its denominator $(D \mathscr{R})$. One finds

$$
\begin{aligned}
& D \mathscr{R}^{\mathrm{Win}}=M^{2}\left(M_{S S} M_{\mathrm{QQ}}-M_{S Q}^{2}\right)^{2}, \\
& D \mathscr{R}^{\mathrm{Rup}}=T M^{2}\left(M_{S S} M_{\mathrm{QQ}}-M_{S Q}^{2}\right)^{2},
\end{aligned}
$$

where $M_{k}=\partial M / \partial k$ and $M_{k j}=\partial^{2} M / \partial k \partial j$.
2.2.2. The Quevedo Metrics. The Quevedo metrics have two kinds with the following forms $[88,89]$ :

$$
d S_{R}^{2}=g_{a b}^{Q} d X^{a} d X^{b}
$$

where $g_{a b}^{Q}$ is

$$
g_{a b}^{\mathrm{Q}}=\Upsilon\left(\begin{array}{cc}
-M_{S S} & 0 \\
0 & M_{\mathrm{QQ}}
\end{array}\right),
$$

with

$$
\Upsilon= \begin{cases}S M_{S}+Q M_{Q}, & \text { Case I } \\ S M_{S}, & \text { Case II. }\end{cases}
$$



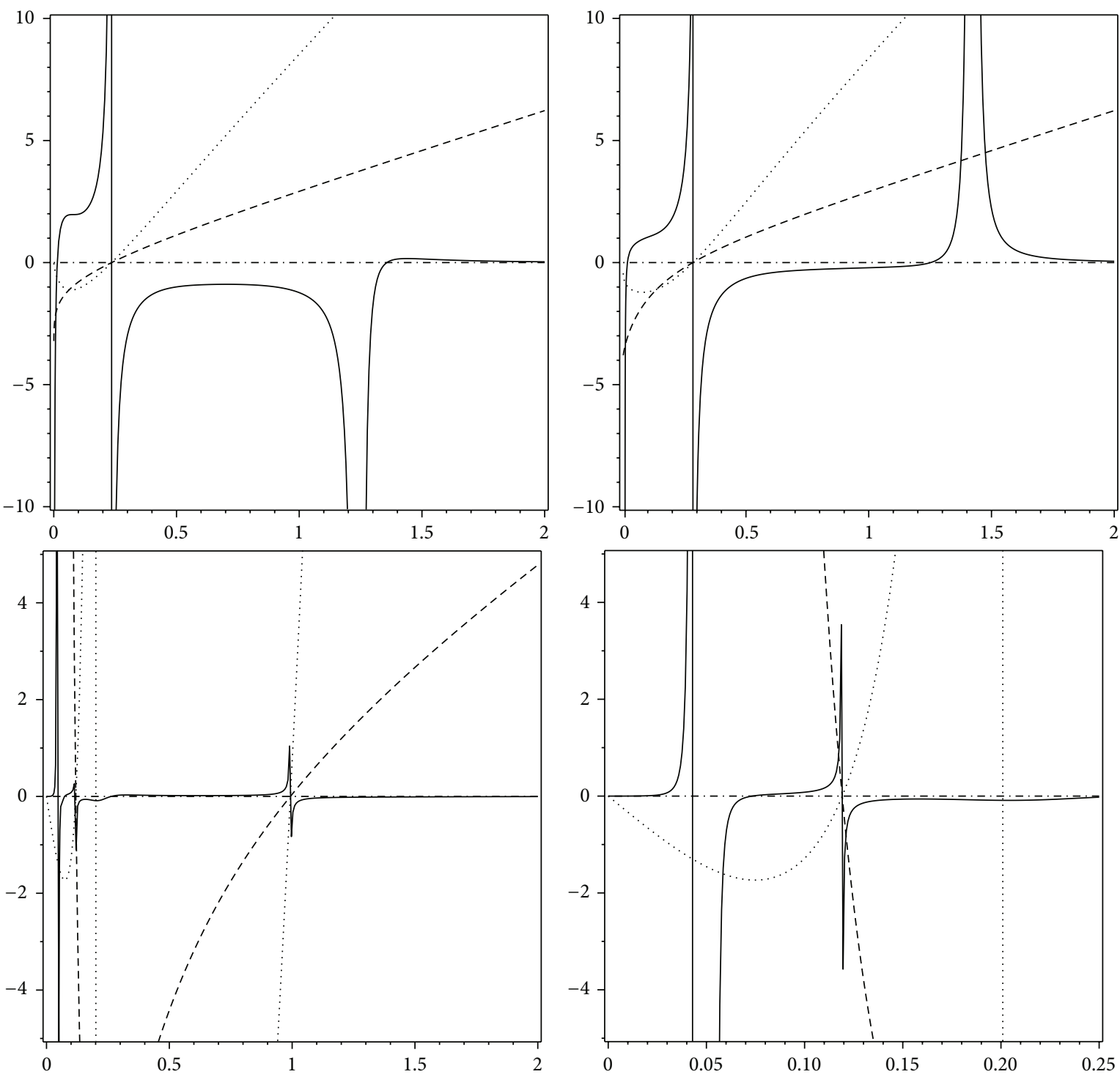

Figure 2: Ruppeiner Ricci scalar (solid line), heat capacity (dotted line), and temperature (dashed line) versus $S$ for $l=1$. HNED model (up-left panel) and SNED model (up-right panel): $q=0.3, \beta=1$. CNED model (down-left panel) and CNED model (down-right panel): $q=1$, $\alpha=0.007$ (different scales).

Taking into account Quevedo metrics, one can find that their related (denomerator of) Ricci scalars can be written as

$$
\begin{aligned}
D \mathscr{R}^{\mathrm{Q}-\mathrm{I}} & =M_{S S}^{2} M_{\mathrm{QQ}}^{2}\left(S M_{S}+Q M_{\mathrm{Q}}\right)^{3}, \\
D \mathscr{R}^{\mathrm{Q}-\mathrm{II}} & =S^{3} M_{S}^{3} M_{S S}^{2} M_{\mathrm{QQ}}^{2} .
\end{aligned}
$$

2.2.3. HPEM Metric. In order to avoid any extra divergencies in TRS which may not coincide with phase transitions of types one and two and also ensure that all the divergencies of the TRS coincide with phase transition points of the both types, HPEM metric was introduced [87]:

$$
g_{a b}=S \frac{M_{S}}{M_{\mathrm{QQ}}^{3}}\left(\begin{array}{cc}
-M_{S S} & 0 \\
0 & M_{\mathrm{QQ}}
\end{array}\right) .
$$

In this case, we have considered the total mass as thermodynamical potential and entropy and electric charge as extensive parameters. Calculations show that denominator of TRS leads to

$$
D \mathscr{R}^{\mathrm{HPEM}}=S^{3} M_{S}^{3} M_{S S}^{2}
$$

\section{The Results of Various Approaches}

Here, we investigate phase transitions of black holes using geometrothermodynamics. For this purpose, we used thermodynamical metrics introduced in previous section for the black holes solutions obtained in Section 2.

For Weinhold metric, none of divergencies of the Ricci scalar coincide with roots of the heat capacity in every theories of NED models that we have considered in this 


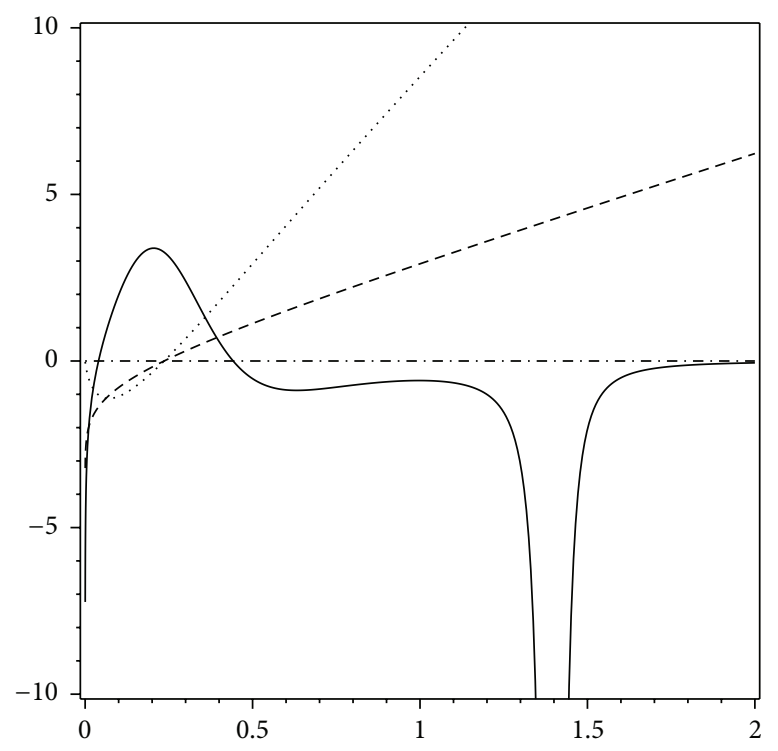

(a)

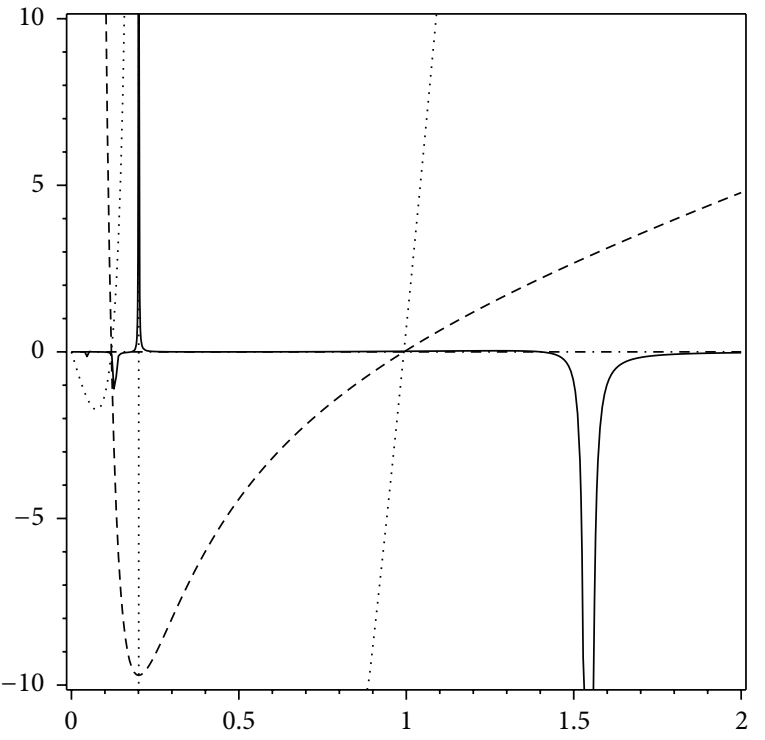

(c)

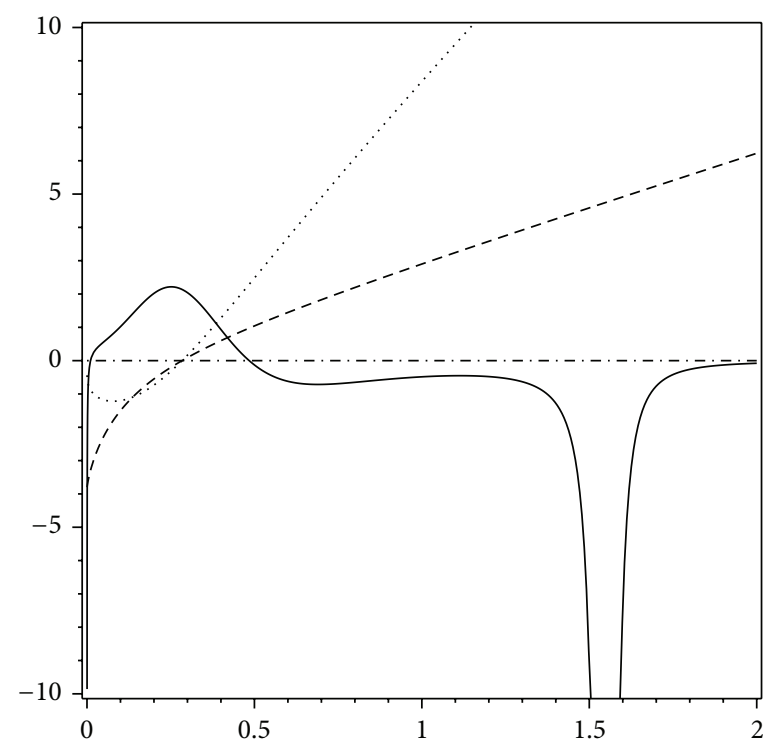

(b)

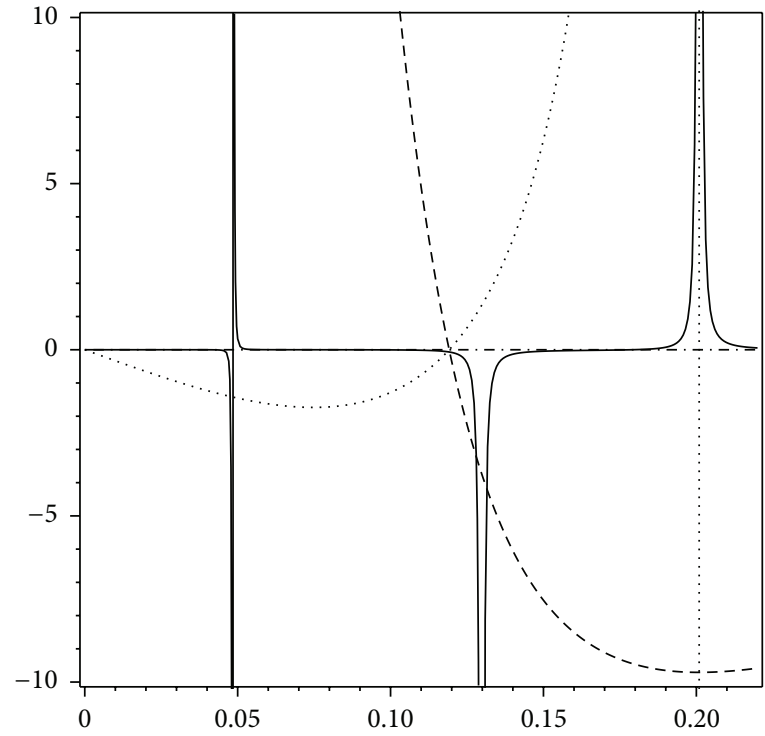

(d)

FIGURE 3: Quevedo Ricci scalar case I (solid line), heat capacity (dotted line), and temperature (dashed line) versus $S$ for $l=1 . H N E D$ model (up-left panel) and SNED model (up-right panel): $q=0.3, \beta=1$. CNED model (down-left panel) and CNED model (down-right panel): $q=1$, $\alpha=0.007$ (different scales).

paper. On the other hand, one of the divergencies of TRS and divergence point of the heat capacity in CNED theory coincide with each other. It is notable that, in cases of the HNED and SNED theories, there is one divergence point for TRS (up panels of Figure 1) whereas, for CNED, there are two divergencies (down panels of Figure 1).

In case of Ruppeiner metric, for HNED model and SNED model, there is a root for heat capacity in which Ricci scalar of the Ruppeiner metric has a divergency. But there is also another divergence point for Ricci scalar which does not coincide with any phase transition point (up panels of Figure 2). Therefore, there is an extra divergence point. In case of the CNED model, two roots and one divergence point are observed for heat capacity in which Ricci scalar has related divergencies. In addition to these divergence points, one extra divergence point is also observed which is not related to any phase transition point (down panels of Figure 2).

As for Quevedo metrics, for case I, similar behavior as Weinhold is observed for all three theories of NED (Figure 3). On the other hand, in case of the other metrics of Quevedo, two divergence points for Ricci scalar were observed for HNED and SNED theories. One of these divergence points coincides with root of the heat capacity for these two nonlinear theories whereas the other one does not (up panels of Figure 4). In case of CNED theory, all the divergence points of TRS coincide with phase transition points except 


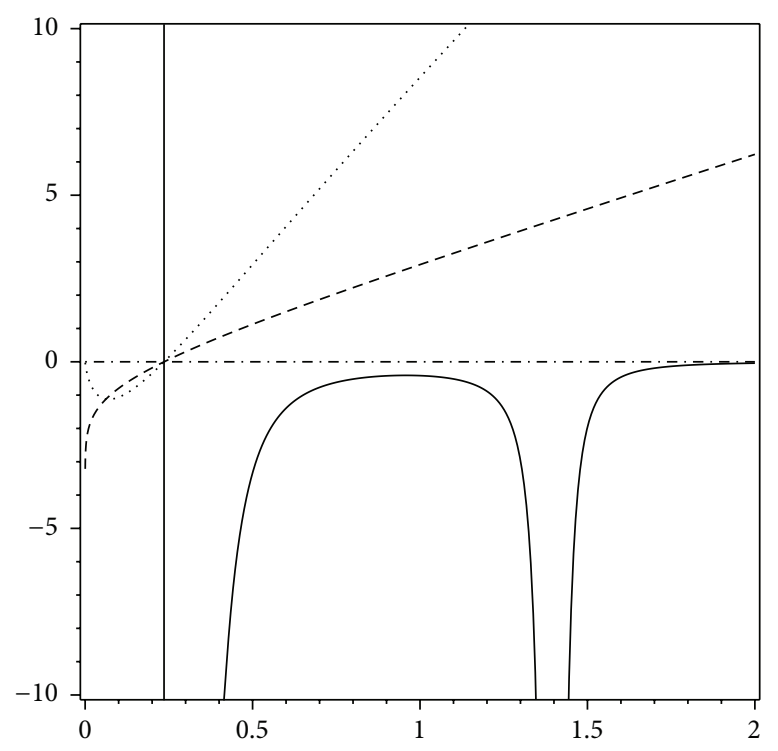

(a)

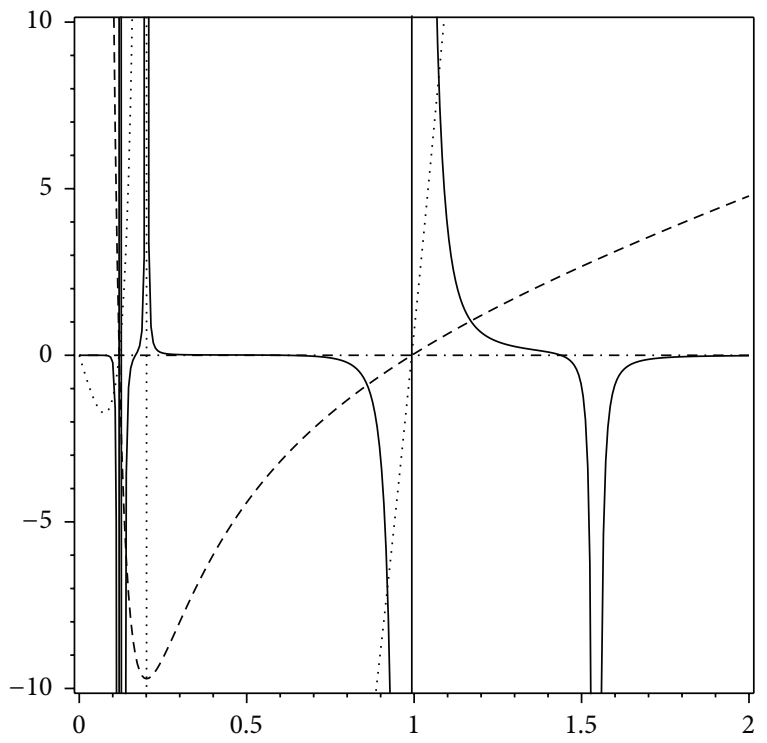

(c)

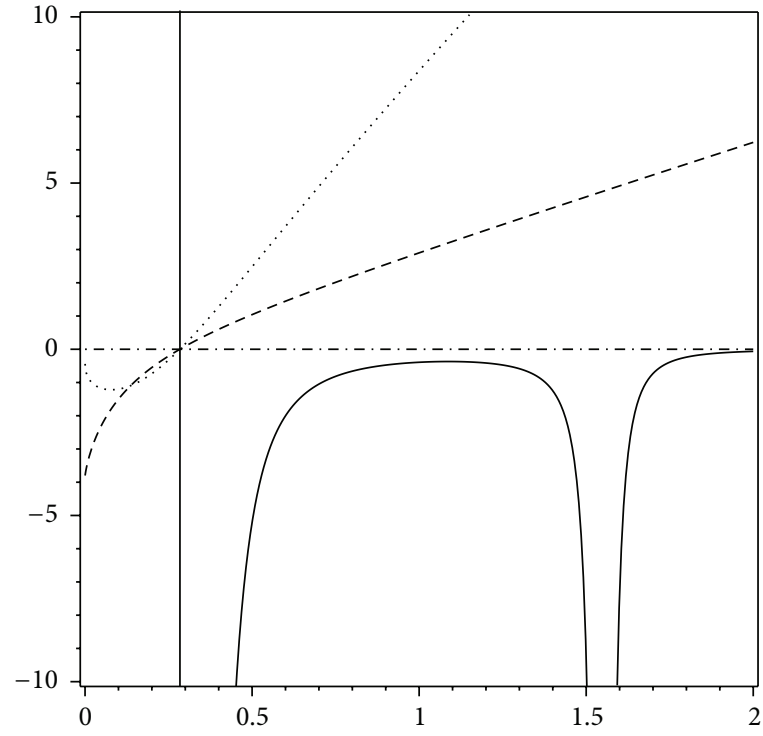

(b)

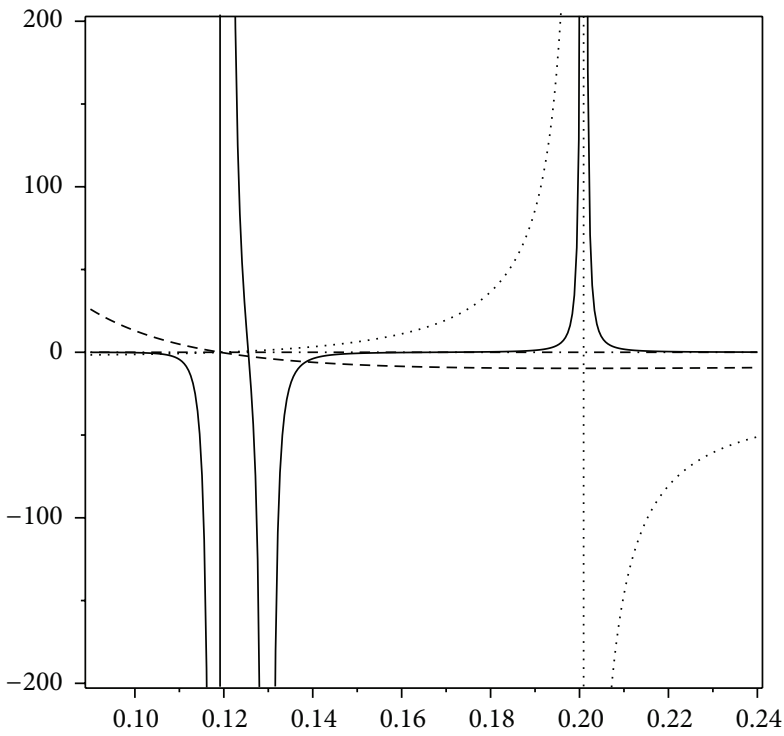

(d)

FIGURE 4: Quevedo Ricci scalar case II (solid line), heat capacity (dotted line), and temperature (dashed line) versus $S$ for $l=1 . H N E D$ model (up-left panel) and SNED model (up-right panel): $q=0.3, \beta=1$. CNED model (down-left panel) and CNED model (down-right panel): $q=1$, $\alpha=0.007$ (different scales).

one (down panels of Figure 4). In other words, Quevedo's metric predicts an extra divergence point, corresponding to the equation $M_{\mathrm{QQ}}=0$, which is not predicted in classical black hole thermodynamics.

It is evident that, in case of HPEM, all types of phase transition points of heat capacity coincide with divergencies of TRS of HPEM method (Figure 5). In other words, independent of the nonlinear theory under consideration, the HPEM method provides a machinery in which no extra divergency for TRS is observed and divergence points of TRS and phase transition points coincide. Another interesting and important property of the HPEM method is the behavior of TRS near divergence point for different types of phase transition. As one can see, the signature and behavior of TRS near divergence point for phase transition type one and two are different. Therefore, independent of studying the heat capacity, one can distinguish these types of phase transition from one another only by studying the behavior of TRS.

\section{Closing Remarks}

In this paper, we have considered BTZ black holes, in the presence of three models of NED. We studied stability and phase transitions related to the heat capacity of the mentioned black holes. Next, we employed geometrical approach to study the thermodynamical behavior of the system. In other 


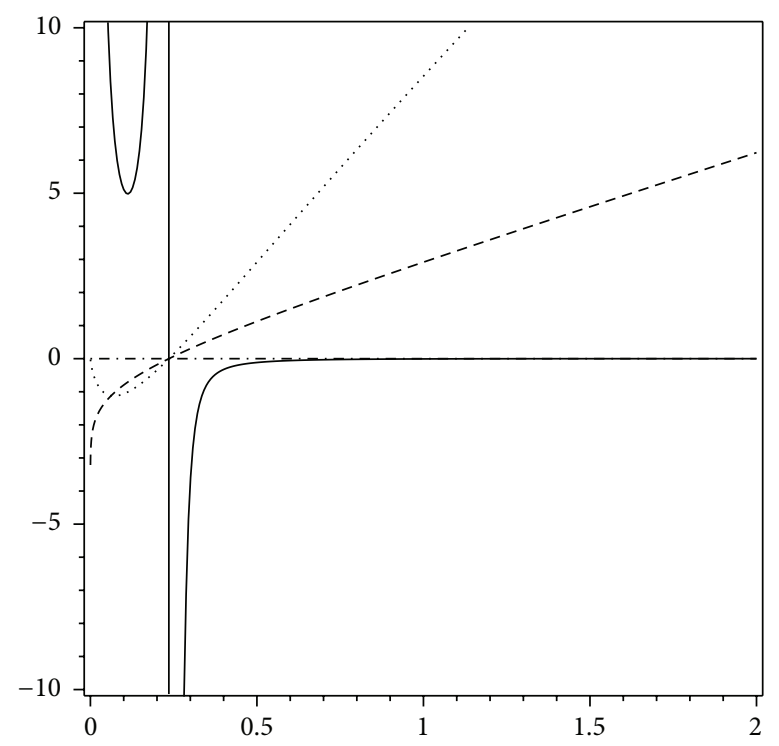

(a)

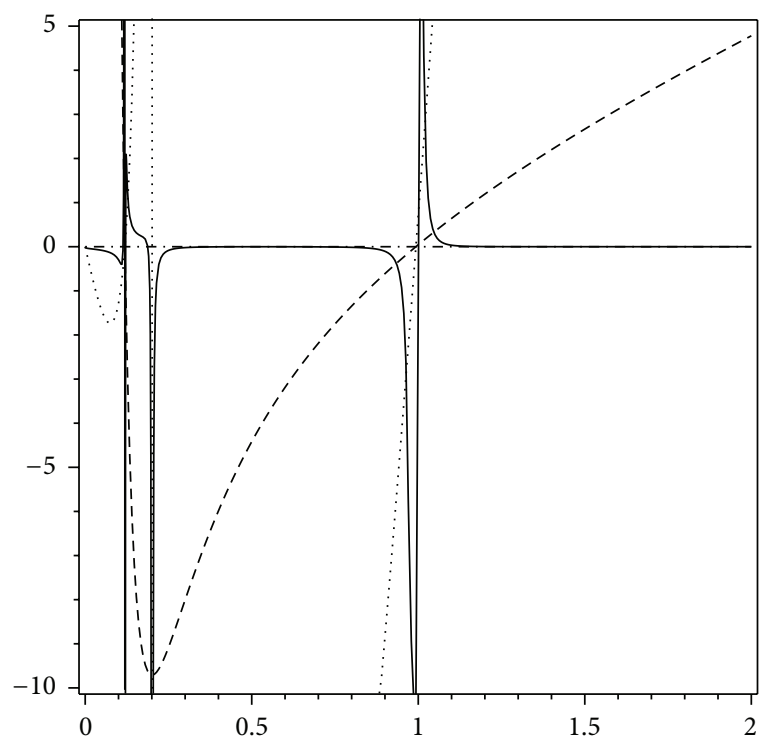

(c)

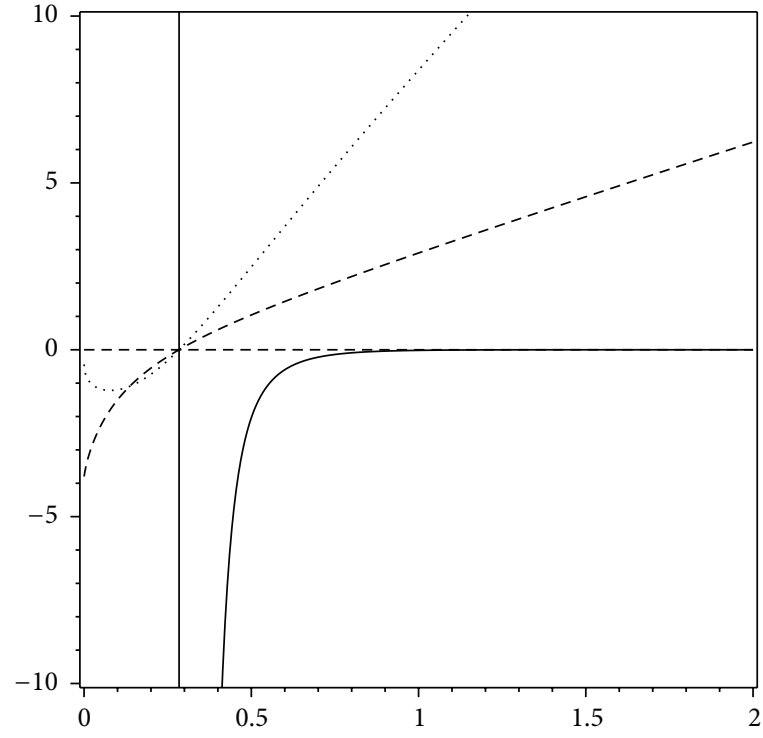

(b)

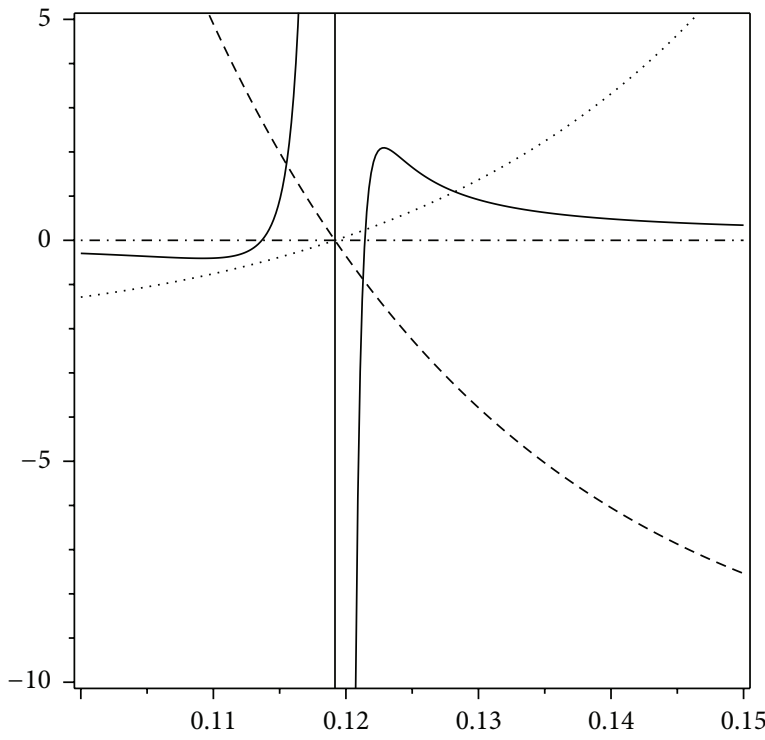

(d)

FIGURE 5: HPEM Ricci scalar (solid line), heat capacity (dotted line), and temperature (dashed line) versus $S$ for $l=1$. HNED model (up-left panel) and SNED model (up-right panel): $q=0.3, \beta=1$. CNED model (down-left panel) and CNED model (down-right panel): $q=1$, $\alpha=0.007$ (different scales).

words, we have studied phase transitions of the system through Weinhold, Ruppeiner, and Quevedo methods. Also, we used the recently proposed approach to study geometrical thermodynamics.

We found that the Weinhold and Ruppeiner metrics for studying these BTZ solutions fail to provide a suitable result. In addition, the divergence points of the Quevedo TRS were not completely matched with the phase transition points of the heat capacity results. In other words, in these approaches, the existence of extra divergencies was observed which were not related to any phase transition point in the classical thermodynamics. In some of these approaches, no divergency of TRS coincided with phase transition points. In order to obtain a consistent results with the classical thermodynamic consequences (the heat capacity), we employed a new thermodynamical metric. In this approach, all the divergencies of TRS coincided with phase transition points. In other words, roots and divergence points of the heat capacity of the BTZ black holes in the presence of each nonlinear models matched with divergencies of TRS of this metric.

Also we found that, in case of HNED and SNED theories, there is no divergency for heat capacity. It means that, like Maxwell theory, these two theories have no second type phase transition. These two nonlinear theories of electrodynamics preserved the characteristic behavior of the Maxwell theory in case of heat capacity. On the other hand, for CNED 
model, two roots and one divergence point were found for the heat capacity. In other words, due to contribution of the nonlinear electromagnetic field, heat capacity enjoys the existence of one more phase transition point of type one and a phase transition of type two. In essence, this theory is a generalization of the Maxwell theory. But this generalization added another property to heat capacity that was not observed for the Maxwell theory.

Finally, it is worthwhile to mention a comment related to Legendre invariancy. It was shown that [98] the Legendre invariance alone is not sufficient to guarantee a unique description of thermodynamical metrics in terms of their curvatures. In addition to Legendre invariancy, one needs to demand curvature invariancy under a change of representation. Therefore, it will be worthwhile to investigate both Legendre and curvature invariancies. In addition, it will be interesting to think about the fundamental relation between the following two issues: (I) agreement of thermodynamical curvature results with usual thermodynamical approaches (such as the heat capacity) and (II) curvature invariancy in addition to the Legendre invariancy. It is also worthwhile to probe the fundamentality of cases (I) and (II) to find that considering which one may lead to satisfy the other one. Although first issue has been investigated for special cases [98], the second one has remained unanalyzed yet. We may address them in an independent work in the future.

\section{Conflict of Interests}

The authors declare that there is no conflict of interests regarding the publication of this paper.

\section{Acknowledgments}

The authors would like to thank the anonymous referee for valuable suggestions. They also thank the Shiraz University Research Council. This work has been supported financially by the Research Institute for Astronomy and Astrophysics of Maragha, Iran.

\section{References}

[1] M. Astorino, "Accelerating black hole in $2+1$ dimensions and $3+1$ black (st)ring," Journal of High Energy Physics, vol. 2011, article 114, 2011.

[2] A. Anabalón, F. Canfora, A. Giacomini, and J. Oliva, "Gribov ambiguity in asymptotically AdS three-dimensional gravity," Physical Review D, vol. 83, no. 6, Article ID 064023, 2011.

[3] L. Hodgkinson and J. Louko, "Static, stationary, and inertial Unruh-DeWitt detectors on the BTZ black hole," Physical Review D, vol. 86, no. 6, Article ID 064031, 16 pages, 2012.

[4] T. Moon and Y. S. Myung, "Rank- $n$ logarithmic conformal field theory in the BTZ black," Physical Review D, vol. 86, Article ID 124042, 2012.

[5] F. Darabi, K. Atazadeh, and A. Rezaei-Aghdam, "Generalized $(2+1)$ dimensional black hole by Noether symmetry," European Physical Journal C, vol. 73, article 2657, 2013.
[6] E. Frodden, M. Geiller, K. Noui, and A. Perez, "Statistical entropy of a BTZ black hole from loop quantum gravity," Journal of High Energy Physics, vol. 2013, no. 5, article 139, 2013.

[7] M. Hassaine, "Rotating AdS black hole stealth solution in $D$ = 3 dimensions," Physical Review D, vol. 89, no. 4, Article ID 044009, 5 pages, 2014.

[8] W. Xu, "Exact black hole formation in three dimensions," Physics Letters B, vol. 738, pp. 472-476, 2014.

[9] B. Wu and W. Xu, "New class of rotating perfect fluid black holes in three dimensional gravity," The European Physical Journal C, vol. 74, article 3007,2014

[10] S. Carlip, “The $(2+1)$-dimensional black hole," Classical and Quantum Gravity, vol. 12, no. 12, article 2853, 1995.

[11] A. Ashtekar, J. Wisniewski, and O. Dreyer, "Isolated horizons in $2+1$ gravity," Advances in Theoretical and Mathematical Physics, vol. 6, pp. 507-555, 2002.

[12] T. Sarkar, G. Sengupta, and B. N. Tiwari, "On the thermodynamic geometry of BTZ black holes," Journal of High Energy Physics, vol. 2006, no. 11, article 015, 2006.

[13] E. Witten, "Anti-de sitter space, thermal phase transition, and confinement in gauge theories," Advances in Theoretical and Mathematical Physics, vol. 2, pp. 505-532, 1998.

[14] S. Carlip, "Conformal field theory, $(2+1)$-dimensional gravity and the BTZ black hole," Classical and Quantum Gravity, vol. 22, no. 12, article R85, 2005.

[15] E. Witten, “Three-dimensional gravity revisited," http://arxiv .org/abs/0706.3359.

[16] M. Banados, C. Teitelboim, and J. Zanelli, "Black hole in threedimensional spacetime," Physical Review Letters, vol. 69, no. 13, pp. 1849-1851, 1992.

[17] M. Bañados, M. Henneaux, C. Teitelboim, and J. Zanelli, "Geometry of the $2+1$ black hole," Physical Review D: Third Series, vol. 48, no. 4, pp. 1506-1525, 1993.

[18] S. Nojiri and S. D. Odintsov, "Can quantum-corrected BTZ black hole anti-evaporate?" Modern Physics Letters A, vol. 13, no. 33, pp. 2695-2704, 1998.

[19] R. Emparan, G. T. Horowitz, and R. C. Myers, "Exact description of black holes on branes II: comparison with BTZ black holes and black strings," Journal of High Energy Physics, vol. 2000, no. 1, article 021, 2000.

[20] S. Hemming, E. Keski-Vakkuri, and P. Kraus, "Strings in the extended BTZ spacetime," Journal of High Energy Physics, vol. 2002, no. 10, article 006, 2002.

[21] M. R. Setare, "Non-rotating BTZ black hole area spectrum from quasi-normal modes," Classical and Quantum Gravity, vol. 21, no. 6, pp. 1453-1457, 2004.

[22] B. Sahoo and A. Sen, "BTZ black hole with Chern-Simons and higher derivative terms," Journal of High Energy Physics, vol. 2006, no. 7, article 008, 2006.

[23] M. Cadoni and M. R. Setare, "Near-horizon limit of the charged BTZ black hole and AdS2 quantum gravity," Journal of High Energy Physics, vol. 2008, no. 7, article 131, 2008.

[24] J. Parsons and S. F. Ross, "Strings in extremal BTZ black holes," Journal of High Energy Physics, vol. 2009, no. 4, article 134, 2009.

[25] E. Ayon-Beato, A. Garbarz, G. Giribet, and M. Hassaine, "Lifshitz black hole in three dimensions," Physical Review D, vol. 80, Article ID 104029, 2009.

[26] O. Gurtug, S. H. Mazharimousavi, and M. Halilsoy, "2+1dimensional electrically charged black holes in Einstein-powerMaxwell theory," Physical Review D, vol. 85, no. 10, Article ID 104004, 2012. 
[27] S. H. Hendi, B. Eslam Panah, and R. Saffari, "Exact solutions of three-dimensional black holes: einstein gravity versus $F(R)$ gravity," International Journal of Modern Physics D, vol. 23, no. 11, Article ID 1450088, 2014.

[28] F. Chen, K. Dasgupta, J. M. Lapan, J. Seo, and R. Tatar, "Gauge/gravity duality in heterotic string theory," Physical Review D: Particles, Fields, Gravitation and Cosmology, vol. 88, no. 6, Article ID 066003, 2013.

[29] M. Fukuma, S. Matsuura, and T. Sakai, "Higher-derivative gravity and the AdS/CFT correspondence," Progress of Theoretical Physics, vol. 105, no. 6, pp. 1017-1044, 2001.

[30] R. Aros, M. Romo, and N. Zamorano, "Conformal gravity from the AdS/CFT mechanism," Physical Review D, vol. 75, no. 6, Article ID 067501, 2007.

[31] E. Ayón-Beato and A. García, "New regular black hole solution from nonlinear electrodynamics," Physics Letters B, vol. 464, no. 1-2, pp. 25-29, 1999.

[32] S. Fernando and D. Krug, "Charged black hole solutions in Einstein-Born-Infeld gravity with a cosmological constant," General Relativity and Gravitation, vol. 35, no. 1, pp. 129-137, 2003.

[33] J. Matyjasek, "Extremal limit of the regular charged black holes in nonlinear electrodynamics," Physical Review D: Third Series, vol. 70, no. 4, Article ID 047504, 2004.

[34] R.-G. Cai, D.-W. Pang, and A. Wang, "Born-Infeld black holes in (A)dS spaces," Physical Review D, vol. 70, no. 12, Article ID 124034, 2004.

[35] T. K. Dey, "Born-Infeld black holes in the presence of a cosmological constant," Physics Letters B, vol. 595, no. 1-4, pp. 484-490, 2004.

[36] S. Fernando, "Thermodynamics of Born-Infeld-anti-de Sitter black holes in the grand canonical ensemble," Physical Review D, vol. 74, no. 10, Article ID 104032, 2006.

[37] M. Hassaïne and C. Martínez, "Higher-dimensional black holes with a conformally invariant Maxwell source," Physical Review D. Particles, Fields, Gravitation, and Cosmology, vol. 75, no. 2, Article ID 027502, 2007.

[38] Y. S. Myung, Y.-W. Kim, and Y.-J. Park, "Thermodynamics and phase transitions in the Born-Infeld-anti-de Sitter black holes," Physical Review D, vol. 78, no. 8, Article ID 084002, 2008.

[39] I.-C. Yang, C.-L. Lin, and I. Radinschi, "The energy of regular black hole in general relativity coupled to nonlinear electrodynamics," International Journal of Theoretical Physics, vol. 48, no. 1, pp. 248-255, 2009.

[40] H. Maeda, M. Hassaine, and C. Martinez, "Lovelock black holes with a nonlinear Maxwell field," Physical Review D, vol. 79, Article ID 044012, 2009.

[41] S. H. Hendi and B. E. Panah, "Thermodynamics of rotating black branes in Gauss-Bonnet-nonlinear Maxwell gravity," Physics Letters B, vol. 684, no. 2-3, pp. 77-84, 2010.

[42] S. H. Hendi, "Rotating black branes in the presence of nonlinear electromagnetic field," The European Physical Journal C, vol. 69, no. 1-2, pp. 281-288, 2010.

[43] O. Mišković and R. Olea, "Conserved charges for black holes in Einstein-Gauss-Bonnet gravity coupled to nonlinear electrodynamics in AdS space," Physical Review D, vol. 83, Article ID 024011, 2011.

[44] P. Li, R.-H. Yue, and D.-C. Zou, "Thermodynamics of third order Lovelock-Born-Infeld black holes," Communications in Theoretical Physics, vol. 56, no. 5, pp. 845-850, 2011.
[45] D. C. Zou, Z. Y. Yang, R. H. Yue, and P. Li, “Thermodynamics of Gauss-Bonnet-BORn-Infeld black holes in AdS space," Modern Physics Letters A, vol. 26, no. 7, pp. 515-529, 2011.

[46] R. Banerjee and D. Roychowdhury, "Critical behavior of BornInfeld AdS black holes in higher dimensions," Physical Review D, vol. 85, no. 10, Article ID 104043, 14 pages, 2012.

[47] S. H. Hendi, "Asymptotic Reissner-Nordström black holes," Annals of Physics, vol. 333, pp. 282-289, 2013.

[48] D.-C. Zou, S.-J. Zhang, and B. Wang, "Critical behavior of BornInfeld AdS black holes in the extended phase space thermodynamics," Physical Review D, vol. 89, Article ID 044002, 2014.

[49] S. H. Mazharimousavi, M. Halilsoy, and O. Gurtug, "A new Einstein-nonlinear electrodynamics solution in $2+1$ dimensions," European Physical Journal C, vol. 74, no. 1, article 2735, 2014.

[50] G. Gibbons, R. Kallosh, and B. Kol, "Moduli, scalar charges, and the first law of black hole thermodynamics," Physical Review Letters, vol. 77, no. 25, pp. 4992-4995, 1996.

[51] N. Bretón, "Smarr's formula for black holes with non-linear electrodynamics," General Relativity and Gravitation, vol. 37, no. 4, pp. 643-650, 2005.

[52] D. Kastor, S. Ray, and J. Traschen, "Enthalpy and the mechanics of AdS black holes," Classical and Quantum Gravity, vol. 26, no. 19, Article ID 195011, 2009.

[53] W. Yi-Huan, "Energy and first law of thermodynamics for Born-Infeld-anti-de-Sitter black hole," Chinese Physics B, vol. 19, no. 9, Article ID 090404, 2010.

[54] B. P. Dolan, "Pressure and volume in the first law of black hole thermodynamics," Classical and Quantum Gravity, vol. 28, no. 23, Article ID 235017, 2011.

[55] D. Kubiznak and R. B. Mann, " $P-V$ criticality of charged AdS black holes," Journal of High Energy Physics, vol. 2012, no. 7, article 33, 2012.

[56] R. G. Cai, L. M. Cao, L. Li, and R. Q. Yang, " $P-V$ criticality in the extended phase space of Gauss-Bonnet black holes in AdS space," Journal of High Energy Physics, vol. 2011, no. 9, article 005, 2011.

[57] S. H. Hendi and M. H. Vahidinia, "Extended phase space thermodynamics and $P-V$ criticality of black holes with a nonlinear source," Physical Review D, vol. 88, no. 8, Article ID 084045, 11 pages, 2013.

[58] M. S. Ma, F. Liu, and R. Zhao, "Continuous phase transition and critical behaviors of 3D black hole with torsion," Classical and Quantum Gravity, vol. 31, no. 9, Article ID 095001, 2014.

[59] J.-X. Mo, X.-X. Zeng, G.-Q. Li, X. Jiang, and W.-B. Liu, "A unified phase transition picture of the charged topological black hole in Hořava-Lifshitz gravity," Journal of High Energy Physics, vol. 2013, article 056, 2013.

[60] R. Tharanath, J. Suresh, and V. C. Kuriakose, "Phase transitions and geometrothermodynamics of regular black holes," General Relativity and Gravitation, vol. 47, article 46, 2015.

[61] S. H. Hendi, S. Panahiyan, and B. Eslam Panah, " $P-V$ criticality and geometrothermodynamics of black holes with Born-Infeld type nonlinear electrodynamics," http://arxiv.org/abs/1410.0352.

[62] J. L. Zhang, R. G. Cai, and H. Yu, "Phase transition and thermodynamical geometry for Schwarzschild AdS black hole in $\mathrm{AdS}_{5} \times \mathrm{S}_{5}$ spacetime," Journal of High Energy Physics, vol. 2015, no. 2, article 143, 2015.

[63] J. L. Zhang, R. G. Cai, and H. Yu, "Phase transition and thermodynamical geometry of Reissner-Nordström-AdS black holes in extended phase space," Physical Review D, vol. 91, no. 4, Article ID 044028, 14 pages, 2015. 
[64] J.-X. Mo and W.-B. Liu, "Phase transitions, geometrothermodynamics, and critical exponents of black holes with conformal anomaly," Advances in High Energy Physics, vol. 2014, Article ID 739454, 10 pages, 2014.

[65] N. Breton and S. E. P. Bergliaffa, "On the stability of black holes with nonlinear electromagnetic fields," http://arxiv.org/abs/ 1402.2922 .

[66] S. Grunau and H. Neumann, "Thermodynamics of a rotating black hole in minimal five-dimensional gauged supergravity," http://xxx.tau.ac.il/abs/1502.06755.

[67] B. P. Dolan, "Thermodynamic stability of asymptotically anti-de Sitter rotating black holes in higher dimensions," Classical and Quantum Gravity, vol. 31, no. 16, Article ID 165011, 23 pages, 2014.

[68] S. H. Hendi and S. Panahiyan, "Thermodynamic instability of topological black holes in Gauss-Bonnet gravity with a generalized electrodynamics," Physical Review D, vol. 90, no. 12, Article ID 124008, 2014.

[69] S. H. Hendi and M. Momennia, "Thermodynamic instability of topological black holes with nonlinear source," The European Physical Journal C, vol. 75, no. 2, 2015.

[70] S. H. Hendi, S. Panahiyan, and R. Mamasani, "Thermodynamic stability of charged BTZ black holes: ensemble dependency problem and its solution," General Relativity and Gravitation, vol. 47, article 91, 2015.

[71] F. Weinhold, "Metric geometry of equilibrium thermodynamics," The Journal of Chemical Physics, vol. 63, no. 6, article 2479, 1975.

[72] F. Weinhold, "Metric geometry of equilibrium thermodynamics. II. Scaling, homogeneity, and generalized Gibbs-Duhem relations," The Journal of Chemical Physics, vol. 63, no. 6, pp. 2484-2487, 1975.

[73] G. Ruppeiner, "Thermodynamics: a Riemannian geometric model," Physical Review A, vol. 20, no. 4, pp. 1608-1613, 1979.

[74] G. Ruppeiner, "Riemannian geometry in thermodynamic fluctuation theory," Reviews of Modern Physics, vol. 67, no. 3, pp. 605-659, 1995.

[75] H. Janyszek, "On the Riemannian metrical structure in the classical statistical equilibrium thermodynamics," Reports on Mathematical Physics, vol. 24, no. 1, pp. 1-10, 1986.

[76] H. Janyszek and R. Mrugała, "Riemannian geometry and the thermodynamics of model magnetic systems," Physical Review A, vol. 39, no. 12, pp. 6515-6523, 1989.

[77] E. J. Brody, "Applications of the Kakutani metric to real-space renormalization," Physical Review Letters, vol. 58, no. 3, pp. 179$182,1987$.

[78] B. P. Dolan, D. A. Johnston, and R. Kenna, "The information geometry of the one-dimensional Potts model," Journal of Physics A. Mathematical and General, vol. 35, no. 43, pp. 90259035, 2002.

[79] W. Janke, D. A. Johnston, and R. Kenna, "Information geometry of the spherical model," Physical Review E, vol. 67, no. 4, Article ID 046106, 4 pages, 2003.

[80] W. Janke, D. A. Johnston, and R. Kenna, "Information geometry and phase transitions," Physica A: Statistical Mechanics and its Applications, vol. 336, no. 1-2, pp. 181-186, 2004.

[81] S. Ferrara, G. W. Gibbons, and R. Kallosh, "Black holes and critical points in moduli space," Nuclear Physics. B, vol. 500, no. 1-3, pp. 75-93, 1997.

[82] R.-G. Cai and J.-H. Cho, "Thermodynamic curvature of the BTZ black hole," Physical Review D, vol. 60, no. 6, Article ID 067502, 4 pages, 1999.
[83] J. E. Åman, I. Bengtsson, and N. Pidokrajt, "Geometry of black hole thermodynamics," General Relativity and Gravitation, vol. 35, no. 10, pp. 1733-1743, 2003.

[84] S. Carlip and S. Vaidya, "Phase transitions and critical behaviour for charged black holes," Classical and Quantum Gravity, vol. 20, no. 16, pp. 3827-3837, 2003.

[85] T. Sarkar, G. Sengupta, and B. N. Tiwari, "On the thermodynamic geometry of BTZ black holes," Journal of High Energy Physics, no. 11, article 015, 20 pages, 2006.

[86] B. Mirza and M. Zamaninasab, "Ruppeiner geometry of RN black holes: flat or curved?" Journal of High Energy Physics, vol. 2007, no. 6, article 059, 2007.

[87] S. H. Hendi, S. Panahiyan, B. E. Panah, and M. Momennia, "A new approach toward geometrical concept of black hole thermodynamics," http://arxiv.org/abs/1506.08092.

[88] H. Quevedo, "Geometrothermodynamics," Journal of Mathematical Physics, vol. 48, no. 1, Article ID 013506, 14 pages, 2007.

[89] H. Quevedo and A. Sánchez, "Geometrothermodynamics of asymptotically Anti-de Sitter black holes," Journal of High Energy Physics, vol. 2008, no. 9, article 034, 2008.

[90] H. Quevedo, A. Sanchez, S. Taj, and A. Vazquez, "Phase transitions in geometrothermodynamics," General Relativity and Gravitation, vol. 43, no. 4, pp. 1153-1165, 2011.

[91] Y. Han and G. Chen, "Thermodynamics, geometrothermodynamics and critical behavior of (2+1)-dimensional black holes," Physics Letters B, vol. 714, no. 2-5, pp. 127-130, 2012.

[92] A. Bravetti, D. Momeni, R. Myrzakulov, and H. Quevedo, "Geometrothermodynamics of higher dimensional black holes," General Relativity and Gravitation, vol. 45, no. 8, pp. 1603-1617, 2013.

[93] A. Bravetti, D. Momeni, R. Myrzakulov, and A. Altaibayeva, "Geometrothermodynamics of Myers-Perry black holes," Advances in High Energy Physics, vol. 2013, Article ID 549808, 11 pages, 2013.

[94] S. H. Hendi, "Thermodynamic properties of asymptotically Reissner-Nordström black holes," Annals of Physics, vol. 346, pp. 42-50, 2014.

[95] S. H. Hendi, "Asymptotic charged BTZ black hole solutions," Journal of High Energy Physics, vol. 2012, no. 3, article 065, 2012.

[96] H. H. Soleng, "Charged black points in general relativity coupled to the logarithmic U(1) gauge theory," Physical Review $D$, vol. 52, no. 10, pp. 6178-6181, 1995.

[97] P. Salamon, J. Nulton, and E. Ihrig, "On the relation between entropy and energy versions of thermodynamic length," The Journal of Chemical Physics, vol. 80, no. 1, pp. 436-437, 1984.

[98] A. Bravetti, C. S. L. Monsalvo, F. Nettel, and H. Quevedo, "The conformal metric structure of Geometrothermodynamics," Journal of Mathematical Physics, vol. 54, Article ID 033513, 2013. 

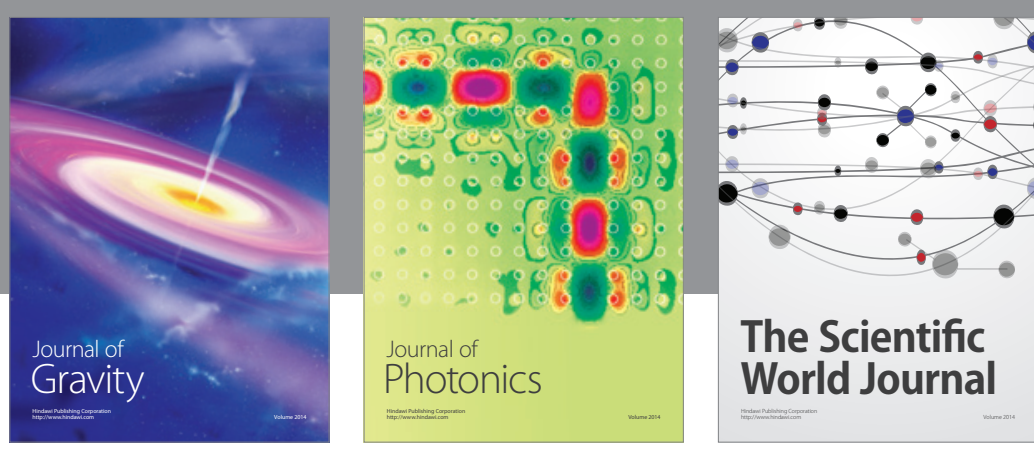

The Scientific World Journal
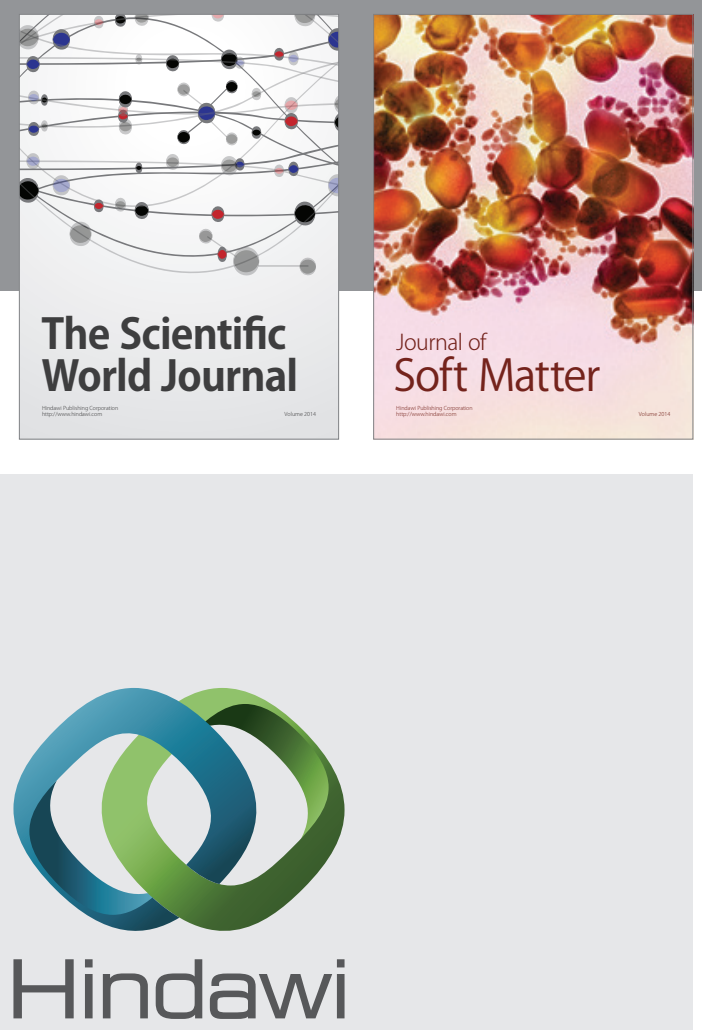

Submit your manuscripts at

http://www.hindawi.com

nternational Journal of

Statistical Mechanics
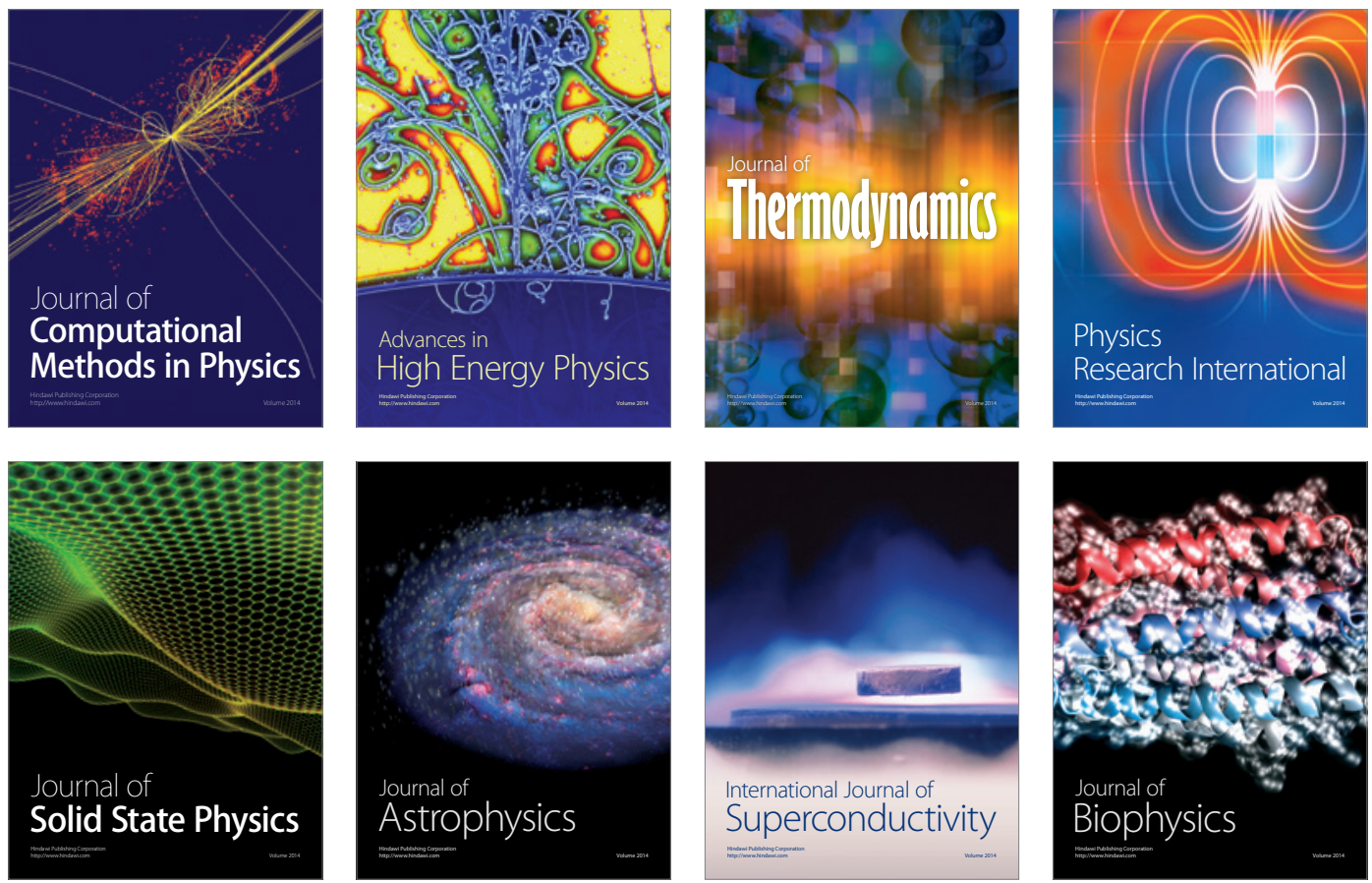
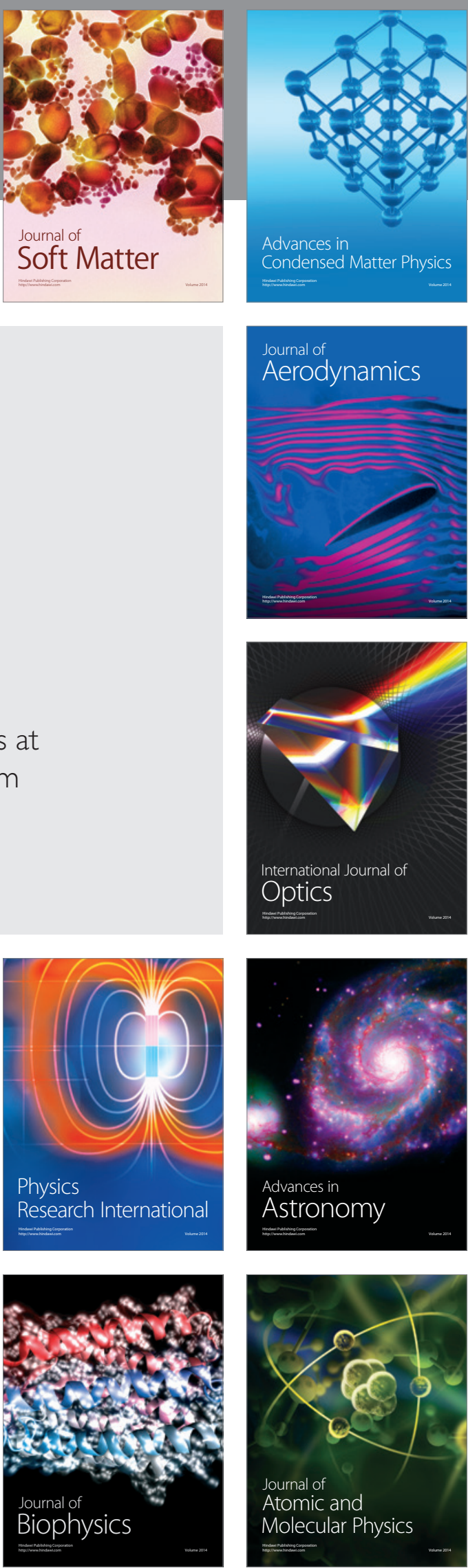